\title{
Impact of Diarrhea after Drinking on Colorectal Tumor Risk: A Case Control Study
}

\author{
Akiko Shiotani ${ }^{1}$, Hideki Ishikawa ${ }^{2}$, Michihiro Mutoh $^{3 *}$, Tatsuya Takeshita ${ }^{4}$, \\ Tomiyo Nakamura ${ }^{5}$, Kanehisa Morimoto ${ }^{6}$, Toshiyuki Sakai ${ }^{2}$, Keiji Wakabayashi ${ }^{7}$, \\ Nariaki Matsuura ${ }^{1}$
}

\begin{abstract}
Background: Recently, the number of colorectal cancer (CRC) cases in Japan has been increasing, and is strongly influenced by alcohol consumption. On the other hand, there are several reports suggesting a relationship between bowel movement (constipation and diarrhea) and CRC development. Moreover, it is generally known that diarrhea may occur after drinking. However, the mechanism by which drinking alcohol increases CRC is not fully clarified yet. We hypothesized that diarrhea after drinking may play an important role in colorectal carcinogenesis. Methods: We examined the presence of diarrhea after drinking and further evaluated the correlation of diarrhea after drinking with the incidence of colorectal tumors. To obtain the status of the feces, a self-recorded questionnaire survey was administered using the dietary-recording method. Blood samples were obtained to analyze the ALDH2 Glu504Lys and ADH1B His 48 Arg polymorphisms. Results: The participants were 417 patients who had undergone a total colonoscopy. The control was selected from 186 patients who underwent a medical checkup at the same hospital during the same time period. The odds ratio for all subjects was 2.1 (95\% CI: 1.18 - 3.80), and that for heavy drinkers was 4.2 (1.48 - 11. 90). Conclusions: The results demonstrated that those who have diarrhea after drinking possess a high risk of developing colon tumors.
\end{abstract}

Keywords: Colorectal cancer- diarrhea- drinking- ADH1B, ALDH2

Asian Pac J Cancer Prev, 20 (3), 795-799

\section{Introduction}

The development of colorectal cancer (CRC) is strongly influenced by environmental factors, including lifestyle. Epidemiological studies have shown strong evidence that an excessive intake of alcohol, lean meat and processed meat are CRC risk factors. On the other hand, the intake of food rich in dietary fiber reduces CRC risk (Institute for Cancer Research, USA, 2007). In Japan, a similar positive relationship has been reported between alcohol consumption and CRC risk (Otani et al., 2003; Shimizu et al., 2003; Wakai et al., 2005). In spite of the evidence, the mechanism by which drinking alcohol increases CRC is not fully clarified yet.

In the case of esophageal cancer, acetaldehyde has been shown to be involved in esophageal carcinogenesis (Yokoyama et al., 2005; Yang et al., 2005; Chen et al., 2006; Yang et al., 2007; Matsuo et al., 2001). Alcohol is oxidized to acetaldehyde mainly by alcohol dehydrogenase (ADH), which consists of $A D H 1 B$ and $A D H 1 C$. Acetaldehyde is further oxidized to acetic acid, mainly by acetaldehyde dehydrogenases (ALDH), such as ALDH2. Certain genetic polymorphisms in $A D H 1 B$ and $A L D H 2$ genes have been characterized regarding their role in defining the metabolizing capacity of these enzymes (Matsuo et al., 2006; Yokoyama et al., 2001). In the case of CRC, however, many published studies have shown that acetaldehyde is not a contributing factor (Yin et al., 2007; Matsuo et al., 2002; Chen et al., 2015). We reported a case-control study on genetic polymorphisms of two enzymes, ADH1B and ALDH2, which have some influence on the development of colorectal adenoma or intramucosal cancer (Shiotani et al., 2015). In this study, we revealed that drinking alcohol increased the risk of colon tumor development in people with high enzyme activity of ADH1B and ALDH2. However, these results did not demonstrate direct explanation why direct drinking promotes CRC development.

${ }^{1}$ Department of Molecular Pathology, Osaka University Graduate School of Medicine and Health Science, ${ }^{6}$ Department of Hygiene and Preventive Medicine, Osaka University Graduate School of Medicine, Osaka, ${ }^{2}$ Department of Molecular-Targeting Cancer Prevention, Kyoto Prefectural University of Medicine, Kyoto, ${ }^{3}$ Epidemiology and Prevention Group, Research Center for Cancer Prevention and Screening, National Cancer Center, Tokyo, ${ }^{4}$ Department of Public Health, Wakayama Medical University School of Medicine, Wakayama, ${ }^{5}$ Faculty of Nutrition, University of Kochi, Kochi, ${ }^{7}$ Graduate Division of Nutritional and Environmental Sciences, University of Shizuoka, Shizuoka, Japan.*For Correspondence: mimutoh@ncc.go.jp 
There are several reports suggesting the relationship between bowel movement and CRC development. A positive relationship has been reported for extremely strong constipation and CRC development (Kotake et al., 1995; Le Marchand et al., 1997; Ghadirian et al., 1998; Jacobs et al., 1998; Roberts et al., 2003). In addition, diarrhea is caused by abnormal bowel movement. However, there are few studies investigating the relationship between diarrhea and CRC development. In those reports, it was reported that the risk of CRC tended to be higher in those with diarrhea (Kojima et al., 2004; Otani et al., 2006; Inoue et al., 1995; Kato et al., 1993). It is generally known that diarrhea may occur after drinking. Thus, we hypothesized that diarrhea after drinking may also play an important role in colorectal carcinogenesis; however, to date, no study has been conducted analyzing CRC patients with diarrhea after drinking and probing the correlation between diarrhea after drinking and CRC development.

Herein, with an aim to address this deficiency, we used our previous case-control study (Ishikawa et al., 2005) to investigate the relationship between the colorectal tumor patients with diarrhea after drinking and the probability of colorectal tumor development. We have examined the presence or absence of diarrhea after drinking and further evaluate the correlation of diarrhea after drinking with the incidence of colorectal tumors. Moreover, we analyzed the relation with colon tumor and genetic polymorphisms of alcohol metabolizing enzymes, depending on the presence or absence of diarrhea after drinking.

\section{Materials and Methods}

\section{Study design and subjects}

The subjects in this study were participants in a randomized clinical trial of the administration of wheat bran and Lactobacillus casei preparation for the prevention of colorectal tumors. The details of this trial have previously been reported (Ishikawa et al., 2005). Briefly, the participants included 417 male and female patients aged 40 to 65 years who had undergone a total colonoscopy at Osaka Medical Center for Cancer and Cardiovascular Diseases, Japan. The subjects with one or more colorectal tumors (early intramucosal tumors and adenomas) subsequently underwent the endoscopic removal of all tumors, and underwent histopathology. The participants were recruited from June 1993 to September 1997. Subjects for whom the genetic polymorphisms of $A D H 1 B$ and $A L D H 2$ were not determined and those with missing values in the correction items were excluded, and the remaining 113 were included in the analysis.

Patients with malignant diseases who underwent intestinal resections, except for appendectomy, as well as those with familial adenomatous polyposis or serious diseases were excluded from both groups.

This study was reviewed and approved by the Ethics Committee of Osaka Medical Center for Cancer and Cardiovascular Diseases. Informed consent was obtained from all the participants in the Case Group and Control Group.

\section{Determining the status of the feces}

The participants in the Case Group and Control Group were interviewed to obtain their height, weight, past medical history, medication history, and family history as well as information on diet, drinking, smoking, lifestyle, physical activity, and the use of non-steroidal antiinflammatory drugs (NSAIDs), such as aspirin. To obtain the status of the feces, a self-recorded questionnaire survey was administered using the dietary-recording method for three consecutive weekdays at the time of entry into the study. In the self-recorded questionnaire, the status of the feces was ascertained according to the following 5 grades: 1) always have diarrhea, 2) sometimes diarrhea, 3) occasionally diarrhea, 4) rarely diarrhea, and 5) do not have diarrhea. For the analysis, 1) and 2) were included into one group "to have diarrhea after drinking" and 3) and 4) to "do not have diarrhea after drinking."

\section{Determining drinking behavior}

Alcohol intake was obtained in terms of the grams of alcohol. A self-recorded questionnaire was also used to collect information on drinking behavior, and nutritionists clarified any unclear statements through interviews. In the self-recorded questionnaire, current drinking behavior was ascertained according to the following 6 grades: 1) drink every day, 2) drink approximately 3-5 times per week, 3) drink approximately 1-2 times per week, 4) drink approximately 1-3 times per month, 5) drink approximately 1-10 times per year, and 6) do not drink. In addition, types of liquor (Japanese sake, beer, Shochu, or whiskey) and amount of liquor (how many sake bottles, large beer bottles, or glasses) they drank were asked to calculate the daily alcohol intake (in grams). We categorized the daily amount of alcohol intake into the following 4 groups: non-drinker, light drinker $(<16 \mathrm{~g} /$ day), moderate drinker ( $\geq 16$ but $<35 \mathrm{~g} /$ day), and heavy drinker ( $\geq 35.0 \mathrm{~g} /$ day $)$.

\section{Measurement of genetic polymorphisms}

Blood samples were obtained from the subjects. We analyzed the ALDH2 Glu504Lys and ADH1B His48Arg polymorphisms. Each of the $A D H 1 B$ and $A L D H 2$ genotypes was determined based on a previously published method (Takeshita et al., 1994; Takeshita et al.,1996). For ALDH2, Glu/Glu was designated as "high", and Glu/Lys and Lys/Lys as "low"; for ADH1B, Arg/His and Arg/Arg as "slow", and His/His as "fast".

\section{Analytical methods}

For sex, height, weight and body mass index (BMI), $\mathrm{t}$ tests were used for comparison between the Case Group and Control Group. Comparisons between the Case Group and Control Group regarding the amount of alcohol intake, smoking and genotype were performed by calculating the odds ratio (OR) and 95\% confidence interval (CI). With regard to the polymorphisms and their combinations in each group, the OR adjusted for age/sex or for age/sex/ $\mathrm{BMI} /$ smoking and the $95 \% \mathrm{CI}$ was calculated using a logistic regression model. IBM SPSS Statistics Ver. 20 was used for the statistical analyses. An unpaired t-test was used to compare the background factors between the 
two groups, and it was regarded as significant with a $\mathrm{p}$ value of $5 \%$ or less.

\section{Results}

Table 1 shows the features of the participants of this study. Men were more frequent in both the Case Group and Control Group. There was a slightly younger trend in the Case Group than the Control Group. There was no difference in height, weight and BMI between the groups. The amount of alcohol consumption was significantly higher in the Case Group. The frequency of genotypes of all polymorphisms was in accordance with Hardy-Weinberg's rule in the Control Group, and the allele frequency was almost consistent with the frequency reported in Japan (Yoshimura et al., 2003).

Tables 2 and 3 show the relationship between the presence of diarrhea after drinking and colorectal tumor. Compared with the Control Group, there are more patients with diarrhea after drinking in the Case Group, even in the examinations using all subjects regardless of non-drinkers or heavy drinkers. Odds ratio for all subjects was 2.1 (95\% confidence interval, CI: $1.18-3.80)$, and for heavy drinkers was 4.2 (1.48 - 11.90). Meanwhile, odds ratio for moderate drinkers was 0.99 (0.38-2.57).

Table 4 shows the relationship between diarrhea after drinking and genetic polymorphisms of ADH $1 \mathrm{~B}$ and ALDH 2 in all subjects, i.e., combined Control and Case Group. The subjects who said diarrhea occurs after drinking mainly harbored ADH 1 B (slow) plus ALDH 2 (high), and the highest ratio was $48.3 \%$, but there was no significant difference.

Table 1. Characteristics of the Study Subjects

\begin{tabular}{lccc}
\hline & $\begin{array}{c}\text { Cases } \\
\text { all }(\mathrm{n}=231)\end{array}$ & $\begin{array}{c}\text { Controls } \\
(\mathrm{n}=186)\end{array}$ & $\begin{array}{c}\text { OR } \\
(95 \% \mathrm{CI})^{\mathrm{b}}\end{array}$ \\
\hline \% Male & 83.5 & 78.5 & \\
Age (yr), mean $\pm \mathrm{SD}$ & $56.4 \pm 6.4^{\mathrm{a}}$ & $58.0 \pm 10.0$ & \\
Height $(\mathrm{cm})$, mean $\pm \mathrm{SD}$ & $164.6 \pm 7.4$ & $164.0 \pm 8.1$ & \\
Weight $(\mathrm{Kg})$, mean $\pm \mathrm{SD}$ & $63.9 \pm 9.6$ & $66.1 \pm 11.0$ & \\
BMI $\left(\mathrm{Kg}^{2}\right)$, mean $\pm \mathrm{SD}$ & $23.5 \pm 3.0$ & $24.5 \pm 3.2$ & \\
Alcohol consumption & & & \\
Non-drinker $(\%)$ & $32(13.9)$ & $65(35.0)$ & 1 \\
Light drinker (\%) & $56(24.2)$ & $40(21.5)$ & 3 \\
Moderate drinker $(\%)$ & $61(26.4)$ & $41(22.0)$ & 3.4 \\
Heavy drinker (\%) & $82(35.5)$ & $40(21.5)$ & 4.6
\end{tabular}

${ }^{\mathrm{a}}$ Significantly different from controls: $\mathrm{P}<0.05 ;{ }^{\mathrm{b}} \mathrm{OR}$, were adjusted for age and sex.

Table 2. Multivariable-Adjusted Odds Ratio for the Presence of Diarrhea after Drinking and Colorecral Tumor in All Subject Including Non- drinker

\begin{tabular}{lcccc}
\hline \multicolumn{5}{c}{ Diarrhea after drinking } \\
& $(-)$ & $(+)(\%)$ & OR & $95 \%$ CI \\
\hline control $(\mathrm{n}=151)$ & 130 & $21(13.9)$ & 1 & \\
case $(\mathrm{n}=216)$ & 157 & $59(27.3)$ & 2.1 & $1.18-3.80$ \\
\hline
\end{tabular}

OR were adjusted for age, sex, BMI and smoking status
Table 3. Multivariable-Adjusted Odds Ratio for the Presence of Diarrhea after Drinking and Colorecral Tumor in Heavy Drinker

Diarrhea after drinking

\begin{tabular}{lcccc} 
& $(-)$ & $(+)(\%)$ & OR & $95 \%$ CI \\
\hline control $(\mathrm{n}=38)$ & 31 & $7(18.4)$ & 1 & \\
case $(\mathrm{n}=75)$ & 42 & $33(44.0)$ & 4.2 & $1.48-11.90$ \\
\hline OR
\end{tabular}

OR were adjusted for age, sex, BMI and smoking status.

Table 4. Ratio of Presence of Diarrhea after Drinking in a Combination of Alcohol Metabolizing Enzyme Activity in All (Case and Control) Group.

\begin{tabular}{lcccc}
\hline $\begin{array}{l}\text { Combination of alcohol } \\
\text { metabolizing enzyme activity }\end{array}$ & $\begin{array}{c}\text { Diarrhea after } \\
\text { drinking } \\
(-)\end{array}$ & OR & $95 \%$ CI \\
\hline $\begin{array}{l}\text { ADH1B (slow). ALDH2 } \\
\text { (low) (n=13) }\end{array}$ & 10 & $3(23.1)$ & 1 & \\
$\begin{array}{l}\text { ADH1B (fast). ALDH2 (low) } \\
(\mathrm{n}=8)\end{array}$ & 6 & $2(25.0)$ & 0.7 & $0.16-2.99$ \\
$\begin{array}{l}\text { ADH1B(slow). ALDH2 } \\
\text { (high) (n=29) }\end{array}$ & 15 & $14(48.3)$ & 2.4 & $0.88-6.52$ \\
$\begin{array}{l}\text { ADH1B (fast). ALDH2(high) } \\
(\mathrm{n}=63)\end{array}$ & 42 & $21(33.3)$ & 1.1 & $0.19-6.20$ \\
\hline $\begin{array}{l}\text { OR were adjusted for age, sex, BMI and smoking status. } \\
\text { (n) }\end{array}$ &
\end{tabular}

\section{Discussion}

This study revealed that those who have diarrhea after drinking possess a high risk of developing colon tumors. Involvement of alcohol metabolizing enzymes (ADH1B, ALDH2) plus diarrhea in the development of colon tumors has not been revealed.

Hitherto, many epidemiological studies have demonstrated that alcohol consumption and colorectal cancer have a positive correlation. However, an acceptable explanation has not been presented. There are several reports of case-control studies that shows a relationship between a history of loose stools / diarrhea and a high risk of CRC. However, there have been no studies showing the relationship between the presence of diarrhea after drinking and colon tumors. Our result that diarrhea after drinking increased the risk of CRC implies that diarrhea after drinking may promote colorectal adenoma and CRC development. In animal experiments, increased production of aberrant prostaglandin E2 production along with bowel movement was suggested to promote the development of colon cancer (Burakoff et al., 1992; Yamaguchi et al., 1991; Kawamori et al., 2003).

An advantage of our study is that the results are not caused by a secondary effect of CRC, because the subjects all had a colorectal tumor removed endoscopically or were from a medical examinee for cancer pick-up. Limitations of our study are as follows: (a) this is a case-control study that may not indicate a reliable causal relationship between diarrhea after drinking and a high risk of developing colon tumors, and (b) the number of cases is relatively small.

Hitherto, several reports showing a relationship between diarrhea and CRC risk have been published (Kojima et al., 2004; Otani et al., 2006; Inoue et al., 1995; Kato et al., 1993). Our study could be misunderstood as 
evaluating the relationship between diarrhea itself and colorectal carcinogenesis. This study showed one of the possibilities that diarrhea caused by alcohol could enhance the risk of CRC. Of course, as the amount of drinking increases, the probability of induction of diarrhea increases. So, it is difficult to evaluate the effects of the amount of drinking or diarrhea itself on colon carcinogenesis separately. Our hypothesis becomes clearer when the risk of $\mathrm{CRC}$ increased in the social drinker with frequent diarrhea after drinking alcohol. However, no significant data were obtained due to the small size of this study. On the other hand, we have to clarify how alcohol affects intestinal flora in subjects who suffer diarrhea from alcohol consumption. Moreover, we do not know whether subjects who are susceptible to diarrhea are likely to suffer diarrhea after drinking alcohol or not. Thus, taking all in to consideration, we cannot divide the diarrhea-CRC axis and alcohol-CRC axis clearly at this moment. Although several issues need to be resolved, our study seems to be worthwhile as it pointed out the issue, the relationship between alcohol-induced diarrhea and an increased risk of CRC.

As far as we know, this is the first report that examined genetic polymorphisms of alcohol metabolizing enzymes (ADH1B, ALDH2) and the presence of diarrhea after drinking alcohol. In this study, subjects with a high activity of ALDH2 and with a low activity of ADH1B tended to have diarrhea after drinking alcohol. In these subjects, it was assumed that the subjects could drink a lot because the metabolism of acetaldehyde was fast, and the blood alcohol concentration in the subjects was high because the alcohol metabolism was slow. Thus, high blood alcohol concentration may affect diarrhea induction. To examine this hypothesis, it is necessary to investigate with an increased number of cases in the future.

In our previous report, drinking alcohol significantly increased the risk of colorectal tumors in the two groups ADH 1 B (slow) plus ALDH 2 (low) and ADH 1 B (fast) plus ALDH 2 (high). To these two high-risk groups, instruction to avoid drinking should be provided in the clinic to reduce CRC risk. We also obtained the data that the patients who possess genetic polymorphisms of ADH 1 B (slow) and ALDH 2 (high) plus diarrhea after drinking tended to have more high risk of CRC than other combinations of genetic polymorphisms we tested. Thus, it is recommended to avoid drinking in the patients who possess ADH $1 \mathrm{~B}$ (slow) and ALDH 2 (high). To conclude, our hypothesis, however, additional examination is required with more number of cases. We are now planning to use a large-scale clinical trial using aspirin (J-CAPP Study II, UMIN000018734) to reveal the role of genetic polymorphisms and defecation status on colorectal carcinogenesis for 1,200 non-aspirin-administered patients and 7,000 aspirin-administered patients.

In summary, the findings from our study suggest that those who have diarrhea after drinking are suggested to possess a high risk of colon tumors. To elucidate the colon carcinogenesis mechanism by drinking alcohol, further examination is needed to reveal the relationship between drinking and diarrhea.

\section{Abbreviations}

$\mathrm{ADH}$, alcohol dehydrogenases; ALDH, aldehyde dehydrogenase; CRC, colorectal cancer; BMI, body mass index: CI, confidence interval; NSAIDs, non-steroidal anti-inflammatory drugs OR, odds ratio.

\section{Funding}

The study is supported by the Grants-in-Aids for the Third-Term Comprehensive 10-Year Strategy for Cancer Control from the Ministry of Health, Labour and Welfare of Japan (H22-3Term-General-014), and the practical research for innovative cancer control from Japan Agency for Medical Research and Development, AMED (JP17ck0106276).

\section{Conflict of Interests}

The authors declare no conflict of interests.

\section{Acknowledgments}

We thank all patients and their families as well as all investigators for their cooperation in this study.

\section{References}

Burakoff R, Percy WH (1992). Studies in vivo and in vitro on effects of PGE2 on colonic motility in rabbits. Am J Physiol, 262, 23-9.

Chen B, Hu KW, Zhang JW, et al (2015). A critical analysis of the relationship between aldehyde dehydrogenases-2 Glu487Lys polymorphism and colorectal cancer susceptibility. Pathol Oncol Res, 21, 727-33.

Chen YJ, Chen C, Wu DC, et al (2006). Interactive effects of lifetime alcohol consumption and alcohol and aldehyde dehydrogenase polymorphisms on esophageal cancer risks. Int J Cancer, 119, 2827-31.

Ghadirian P, Maisonneuve P, Perret C, et al (1998). Epidemiology of sociodemograpphic characteristics, lifestyle, medical history, and colon cancer: a case-control study among French Canadians in Montreal. Cancer Detect Prev, 22, 396-404.

Inoue M, Tajima K, Hamajima N, et al (1995). Subsite-specific risk factors for colorectal cancer:A hospital-based case-control study in Japan. Cancer Causes Control, 6, 14-22.

Ishikawa H, Akedo I, Otani T, et al (2005). Randomized trial of dietary fiber and Lactobacillus casei administration for prevention of colorectal tumors. Int J Cancer, 116, 762-7.

Jacobs EJ, White E (1998). Constipation, laxative use, and colon cancer among middle-aged adults. Epidemiology, 9, 385-91.

Kato I, Tominaga S, Matsuura A, et al (1993). Case-control study of bowel habits and colorectal adenoma and cancer. $J$ Epidemiol, 3, 1-5.

Kawamori T, Uchiya N, Sugimura T, et al (2003). Enhancement of colon carcinogenesis by prostaglandin E2 administration. Carcinogenesis, 24, 985-90.

Kojima M, Wakai K, Tokudome S, et al (2004). Bowel movement frequency and risk of colorectal cancer in a large cohort study of Japanese men and women. Br J Cancer, 90, 1397-401.

Kotake K, Koyama Y, Nasu J, et al (1995). Relation of family history of cancer environmental factors to the risk of colorectal cancer:a case-control study. Jpn J Clin Oncol, 25, 195-202.

Le Marchand L, Wilkens LR, Kolonel LN, et al (1997). Association of sedentary lifestyle, obesity, smoking, alcohol 
use, and diabetes with the risk of colorectal cancer. Cancer Res, 57, 4787-94.

Matsuo K, Hamajima N, Hirai T, et al (2002). Aldehyde dehydrogenase 2 (ALDH2) genotype affects rectal cancer susceptibility due to alcohol consumption. J Epidemiol, 12, 70-6.

Matsuo K, Hamajima N, Shinoda M, etal(2001). Gene-environment interaction between an aldehyde dehydrogenase-2 (ALDH2) polymorphism and alcohol consumption for the risk of esophageal cancer. Carcinogenesis, 22, 913-6.

Matsuo K, Wakai K, Hirose K, et al (2006). Alcohol dehydrogenase 2 His47Arg polymorphism influences drinking habit independently of aldehyde dehydrogenase 2 Glu487Lys polymorphism: Analysis of 2,299 Japanese subjects. Cancer Epidemiol Biomarkers Prev, 15, 1009-13.

Otani T, Iwasaki M, Inoue M, et al (2006). Bowel movement, state of stool, and subsequent risk for colorectal cancer: The Japan Public Health Center-Based Prospective Study. Ann Epidemiol, 16, 888-94.

Otani T, Iwasaki M, Yamamoto S, et al (2003). Alcohol consumption,smoking, and subsequent risk of colorectal cancer in middle-aged and elderly Japanese men and women: Japan Public Health Center-based Prospective Study. Cancer Epideol Biomark Prev, 12, 1492-500.

Roberts MC, Millikan RC, Galanko JA, et al (2003). Constipation, laxative use, and colon cancer in a North Carolina population. Am J Gastroenterol, 98, 857-64.

Shimizu N, Nagata C, Kametani M, et al (2003). Height, weight, and alcohol consumption in relation to the risk of colorectal cancer in Japan: a prospective study. Br J Cancer, 88, 1038-43.

Shiotani A, Ishikawa H, Mutoh M, et al (2015). Genetic polymorphisms in ADH1B and ALDH2 are associated with colorectal tumors in Japan: Case-control study. $J$ Cancer Therapy, 6, 1054-62.

Takeshita T, Mao XQ, Morimoto K (1996). The contribution of polymorphism in the alcohol dehydrogenase beta subunit to alcohol sensitivity in a Japanese population. Hum Genet, 97, 409-13.

Takeshita T, Morimoto K, Mao X, et al (1994). Characterization of the three genotypes of low Km aldehyde dehydrogenase in a Japanese population. Hum Genet, 94, 217-23.

Wakai K, Kojima M, Tamakoshi K, et al (2005). Alcohol consumption and colorectal cancer risk: findings from the JACC Study. J Epidemiol, 15, 173-9.

World Cancer Research Fund and American Institute for Cancer Research: American-Institute for Cancer Research, 2007.

Yamaguchi A, Nishimura G, Katoh M, et al (1991). Investigation of colonic prostaglandins in carcinogenesis in the rat colon. Dis Colon Rectum, 34, 572-6.

Yang CX, Matsuo K, Ito H, et al (2005). Esophageal cancer risk by ALDH2 and ADH2 polymorphisms and alcohol consumption: Exploration of gene-environment and gene-gene interactions. Asian Pac J Cancer Prev, 6, 256-62.

Yang SJ, Wang HY, Li XQ, et al (2007). Genetic polymorphisms of ADH2 and ALDH2 association with esophageal cancer risk in southwest China. World J Gastroenterol, 13, 5760-4.

Yin G, Kono S, Toyomura K, et al (2007) Alcohol dehydrogenase and aldehyde dehydrogenase polymorphisms and colorectal cancer: the Fukuoka colorectal cancer study. Cancer Sci, 98, 1248-53.

Yokoyama A, Kato H, Yokoyama T, et al (2005). Genetic polymorphisms of alcohol and aldehyde dehydrogenases and glutathione S-transferase M1 and drinking, smoking, and diet in Japanese men with esophageal squamous cell carcinoma. Carcinogenesis, 23, 1851-9.

Yokoyama A, Muramatsu T, Omori T, et al (2001). Alcohol and aldehyde dehydrogenase gene polymorphisms and oropharyngolaryngeal, esophageal and stomach cancers in Japanese alcoholics. Carcinogenesis, 22, 433-9.

Yoshimura K, Hanaoka T, Ohnami S, et al (2003). Allele frequencies of single nucleotide polymorphisms (SNPs) in 40 candidate genes for gene-environment studies on cancer: data from population-based Japanese random samples. J Hum Genet, 48, 654-8.

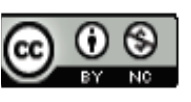

This work is licensed under a Creative Commons AttributionNon Commercial 4.0 International License. 